\title{
Evaluation of the Microcirculation on Perfusion-Phase Analysis of Ultrasound Contrast Echocardiography Using Intuitive and Easy-to-Use Software Tools
}

\author{
JA Morgado-Migueles ${ }^{1}$, DR García-Sánchez ${ }^{1}$, D Moratal-Pérez ${ }^{1}$, J Millet-Roig ${ }^{1}$, \\ V Bodí-Peris ${ }^{2}$, FJ Chorro-Gascó ${ }^{2}$ \\ ${ }^{1}$ Universidad Politécnica de Valencia, Valencia, Spain \\ ${ }^{2}$ Hospital Clínico Universitario de Valencia, Valencia, Spain
}

\begin{abstract}
The principal idea of these software tools is to endow the cardiologists with an appropriate ability covering all their necessities, looking for the detection and analyze of the interesting regions of the myocardium under consideration. To be exactly, the study of the microcirculation in the walls of the myocardium lead us to the analysis of ultrasound contrast echocardiography on perfusion phase after the administration of the adequate contrast agent.

The specialists have enough and complete instrumentation to get myocardial contrast echocardiography, but the possibility of analysing is reduced. There are several techniques to detect the infarction area, but the facility to quantify its level of damage (necrosis) by specific data is still limited nowadays. These programs offer the user a complete capacity to display different parameters on perfusion images. This is the lack that cover the software presented in this article together with the results obtained using them during the evaluation of the microvasculation.
\end{abstract}

\section{Introduction}

Nowadays, contrast myocardial echocardiography has been proposed as the most suitable technique to examine the microvasculation on an infracted myocardium, studying the ability of the left ventricle walls to absorb the contrast agent supplied [1].

The failure of absorbing leads us to the necrosis because of the obstruction of the coronary artery. It is essential to open it using appropriate thrombolytic medication and also angioplasty. To get a fitting diagnosis of the microcirculation is decisive a reliable regional myocardial perfusion assessment. There are two options: intracoronary or intravenous myocardial contrast echocardiography, based on the large acoustical impedance between ultrasound contrast agents injected through a coronary catheter and the blood by itself [2-4].
After this administration, the walls of the left ventricle arrive to the perfusion phase. A thoroughly study of this variation of the opacification allows the cardiologists to define an ischemic heart attack [5-6].

Then, the doctors have several methods of cardiac image registration [7] where had been developed several detection techniques to evaluate on real-time this myocardial contrast enhancement [1][8-10]. Anyway, the cardiologists only use the actual medical instrumentation to describe the microvasculation on the walls of the myocardium. They get a subjective and qualitative analysis. That is the reason why the implementation of these software called Perfusion Echum (P-Echum) and ICARO is crucial for searching a more objective and quantitative valuing.

\section{Methods}

We faced the creation and development of P-Echum and ICARO taking a great advantage of the powerful ability of calculation of MATLAB 6.5 (The Mathworks Inc., Natick, MA, USA). Because of its friendly graphical user interface (GUI), we also guarantee the user easy-touse computer software.

The Service of Cardiology of the University Clinic Hospital of Valencia (Spain) kindly provides the perfusion videos used by both programs. These videos were digitally taken from Agilent SONOS 5500 Echocardiograph (PHILIPS) after contrast agent administration called SonoVue [5-6].

After reviewing several analysis methods on real time [7-11] and defining the requirements of P-Echum, we start the implementation of this software by checking segmentation techniques to automatize the selection of the different regions [1][8][11]. The final option was to offer the user all the control about all the regions and zones, just to display different parameters like area, intensity, percentage ratios among others.

By the other hand, the Video Editor integrated in MATLAB 6.5 allows the automatic creation of perfusion 


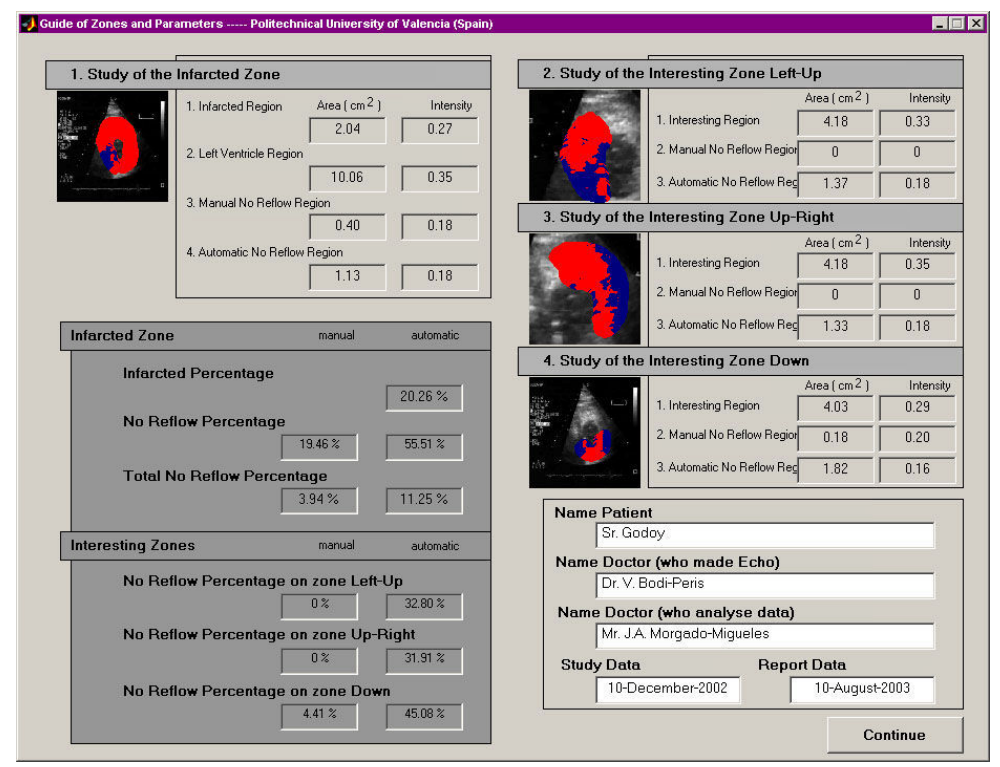

Figure 1 - Perfusion Echum principal guide window.

sequences from a real time video, and also extraction of specific frames from the same video. Using this Editor, $I C A R O$ analyze the contrast and tiger-intensity capture.

\section{Results}

The study of the microcirculation on the walls of the left ventricle requires a very exhaustive analysis of the perfusion images. Specifically, the cardiologists deal with two different myocardial contrast echocardiography according to its contrast administration (intracoronary -ic- and intravenous -iv-) and also its comparison. Being much more specific, 80 ic perfusion studies and 60 iv perfusion studies (all real cases) have been taken into account, with a quantification study time from 5 to 15 minutes.

Before presenting the results obtained by this study, we show both softwares with the options offered to the user.

\subsection{Perfusion Echum}

Perfusion Echum offers the cardiologist the option to analyse the infracted zone, and also three interesting zones where the user can analyse any plane (two cavities, four cavities) and any level (basal, mid, apical). From any zone, the program gives different parameters like area or intensity mean, and also several percentage ratios.

The study of the infracted zone means the first step to carry out an appropriate analysis of the microvasculation. The user should choose the suitable frame from the perfusion video where we focus this study. After that, it is important to access to the calibration phase (the user can define the relationship between pixels and centimetres on the perfusion image, and also the intensity mean as a maximum reference on the same frame).

On the principal window, the cardiologist can begin the manually selection of four regions: 1. Infarcted region, 2. Left ventricle region, 3. Manual no reflow region, and 4. Automatic no reflow region. The last one would be calculated by the program thanks to a no reflow threshold defined by the user. After de selection of these regions, $P$-Echum displays the parameters of area (centimetres square) and intensity mean (adimensonal) of every one of them. We can also see different percentage ratios defined by default: infarction percentage, manual no reflow percentage, automatic no reflow percentage, manual total no reflow percentage, and automatic total no reflow percentage.

The second part of the analysis of the microcirculation takes the specialist to the study of almost three interesting zones. On these windows, we can select another perfusion echocardiographic video depending on the selected plane or/and section of the myocardium. In any case, it is important not to forget the calibration phase to describe 


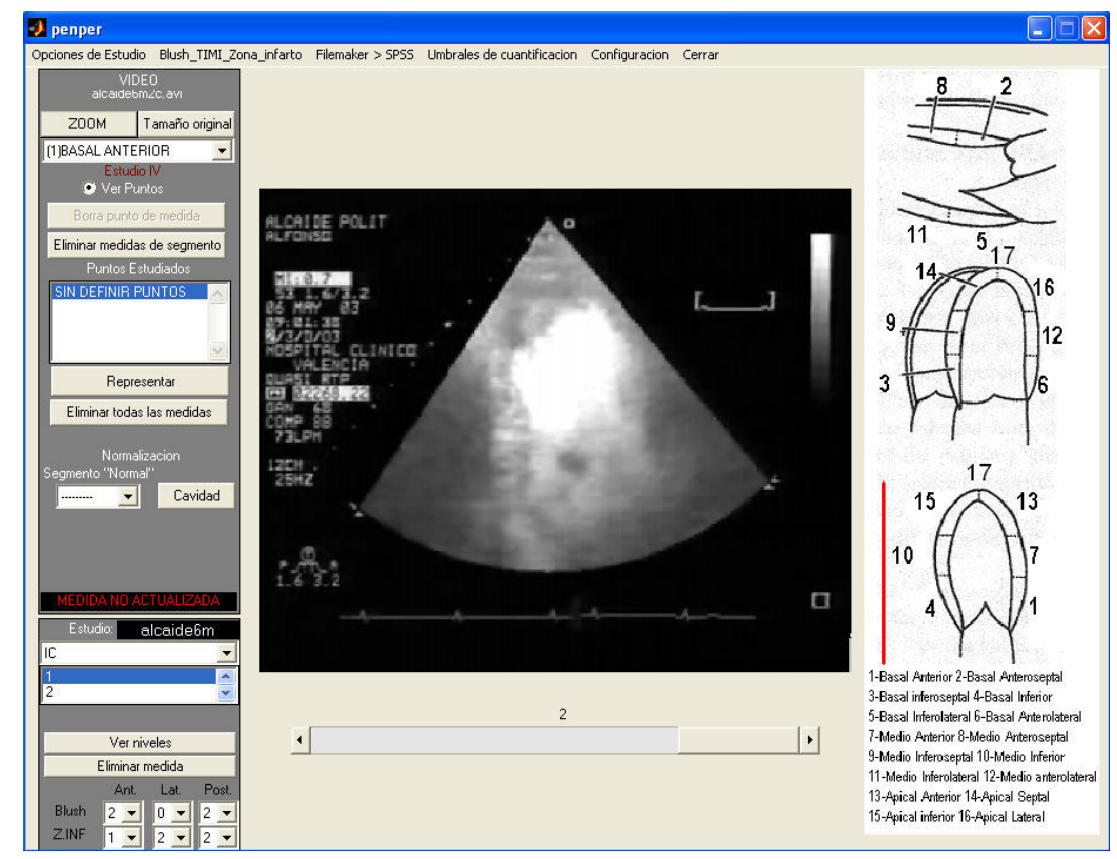

Figure 2 - ICARO main selection window.

the real dimensions of the perfusion image.

Now, the user only can pick up three regions: 1 . Interesting region, 2. Manual no reflow region, and 3. Automatic no reflow region. The last one would be drawn by the program again, and the other regions would be established by the cardiologist. After the selection of these regions, would be displayed their parameters of area and intensity mean and also some by default percentage ratios: manual no reflow percentage, and automatic no reflow percentage.

After the study of these zones, the program present all the parameters of any region of any zone and also the by default percentage ratios (see figure 1). It is possible to store these numeric and personal data on a file, using different formats. In general, P-Echum creates a web file (html) and also an image file (bmp, fig). The user can also print by printer this same window, and the rest of the windows where the study had been taken placed.

\subsection{ICARO}

ICARO completes the analysis of the microcirculation calculating contrast raising parameters, like the triggerintensity valuing and the myocardial flow valuing. From ic and iv myocardial contrast echocardiography, it is possible to evaluate the intensity level in every segment, offering a normalized quantification of these levels. The analysis of the first-step perfusion sequences of myocardial contrast echocardiography allows the calculation of the most important parameters of the raising curve in every segment, emphasizing the maximum plateau level, the raising slope, and the product between both valuing [12]. The evaluation of these characteristic parameters will be taken into account following three different views (long paraexternal, four cameras, and two cameras) [13].

The results of the analysis, such as by segments (17 segments) as by territories (anterior, lateral and posterior) will be resumed in a report document using web format, containing text, video and images. ICARO allows to import and to export these results automatically such as to data bases as to statistical analysis programs, making more easy the following of the patients and the elaboration of statistical studies with an elevate number of real cases.

In figure 2, we can observe the main window of ICARO. According to the segments and the territories (defined on the right side of the window), it is possible to obtain different parameters after selecting the point where the user wants to evaluate the behaviour of the contrast agent, for example (on the echocardiography image). Later, the results obtained from this analysis will be resumed in the adequate report.

\section{Discussion and conclusions}

Both software programs have the significant objective to endow the cardiologists a crucial ability to analyze any myocardial contrast echocardiography. The idea is to offer the user objective and quantitative valuing of the microcirculation on the walls of the left ventricle, looking for several interesting parameters and percentage ratios. 
Remember that the actual medical instrumentation only gives a subjective and qualitative valuing of the ischemic myocardium.

We have to point out on the great handling of these softwares because of their great adaptability to the user's necessities and also to his/her knowledge about computer systems. In that way, both programs suppose an easy-touse tool thanks to the very complete help menu.

Starting from the situation described lines before, the specialist use $P$-Echum and ICARO to get some interesting results. The well-diagnosed cases using iv method suppose the $95 \%$ (98\% using ic method) of the $80 \%$ no-reflow cases detected by the reference method (ic method). It is important to point out that the main technique used in many hospitals as the most adequate is the last one (reference method). In this same direction, the use of trigger images is more correct than myocardial flow to detect the normal behaviour of the ischemic area, concluding this first technique as a reliable and easy one to an adequate analyse.

We can define a serious ischemic myocardium infarct under the $25-30 \%$ of the reference intensity mean selected by the user on the calibration phase corresponding to the reflow region on the walls of the left ventricle. It means that more than the $50 \%$ of the infarcted area described by the cardiologist manually represents the real no-reflow area (all the cases under consideration have infarcted percentage ratios between $30 \%$ and $40 \%$-important heart attack-). After a thorough pursuit of these clinical cases during six months, and thanks to the appropriate medication, we can point out the reduction of the infarcted percentage ratio on a $40-50 \%$ depending of every case.

In conclusion, $P$-Echum and ICARO have been totally created and developed by the BET Research Group of the Universidad Politécnica of Valencia and have been satisfactorily tested on the Cardiology Department of the Hospital Clinico Universitario of Valencia for six months. The results obtained during the evaluation of the microvasculation using both software programs for both contrast analysis methods confirm the advantages of one metrod (iv) over the other one (ic), specially in relation with the patients and their comfort.

\section{Acknowledgements}

This work was partly supported by CTIDIA/202/053 and "Programa Incentivo Investigación UPV".

Special thanks to those people who made Perfusion Echum and ICARO possible.

\section{References}

[1] Malpica N, Santos A, Pérez E, García MA, Desco M. Reposicionamiento de regiones de interés en secuencias de ecocardiografía de contraste. In CASEIB 2002: Medical
Imaging, Proceedings, pp. 121-124.

[2] Sieswerda GT, Kamp O. The promise of myocardial contrast echocardiography for clinical cardiology. EMEXcardio.org (www.emex.nl). 2001.

[3] Linder JR, Villanueva FS, Dent JM. Assessment of resting perfusion with myocardial contrast echocardiography: theoretical and practical consideration. American Heart Journal. 2000. Vol. 139, pp. 231-240.

[4] Tei C, Sakamaki T, Shah P. Myocardial contrast echocardiography: a reproducible technique of myocardial opacification for identifying regional perfusion defects. Circulation. 1983. Vol. 67, pp. 585-593.

[5] Lindner JR, Ismail S, Spotnitz WD, Skyba DM, Goodman NC, Kaul S. Albumin microbubble persistence during myocardial contrast echocardiography is associated with microvascular endothelial glycocalyx damage. Circulation. 1998. pp. 2187-2194.

[6] Borrayo G, Careaga G, Muro CJ, Autrey A, Pérez P, Yañez R, Espínola G, Argüero R. Assessment of right ventricular function using contrast echocardiography in patients with myocardial infarction. Revista Española de Cardiología. 2003. Vol. 56 (2), pp. 175-180.

[7] Mäkela T, Katila T. A review of cardiac image registration methods. IEEE Transactions on Medical Imaging. 2002. Vol. 21 (9), pp.1011-1021.

[8] Song M, Haralick RM, Sheehan FH, Johnson RK. Integrated surface model optimisation for freehand threedimensional echocardiography. IEEE Transactions on Medical Imaging. 2002. Vol. 21 (9), pp. 1077-1090.

[9] De Jong N. Imaging methods for myocardial contrast echocardiography: what to expect in the near future. European Journal of Echocardiography. 2002. Vol. 3 (4), pp. 248-249.

[10] Song M. Ultrasound imaging simulation and echocardiographic image synthesis. Master's Thesis. Department of Electrical Engineering, University of Washington, Seattle. 1999.

[11] Canny J. A computational approach to edge detection. IEEE Transactions Pattern Analysing Machina Intell. 1986. Vol. PAMI-8, pp. 679-698.

[12] Wei K, Linka AZ, Sklenar J, Jayaweera AR, Skyba DM, Kaul S. Assessment of transmural distribution of myocardial perfusion with contrast echocardiography. Circulation. 1998. Vol. 98, pp. 1912-1920.

[13] Cerqueira MD, Weissman NJ, Dilsizian V, Jacobs AK, Kaul S, Laskey WK, Pennell DJ, Rumberger JA, Ryan T, Verani MS. Standardized myocardial segmentation and nomenclatura for tomographic imaging of the heart. Circulation. 2002. Vol. 105 (4), pp. 539-542.

Address for correspondence.

José Antonio Morgado-Migueles

Dpto. Ingeniería Electrónica

ETSI Telecomunicación - UPV

Camino de Vera, s/n

46022 Valencia

SPAIN

e-mail: jomormi@upvnet.upv.es 